\title{
In situ probing of stress-induced nanoparticle dispersion and friction reduction in lubricating grease
}

\begin{abstract}
Particulate additives improve the lubricating performance of oils and greases. Effects of nanoparticles on lubrication are not well understood because it is not possible to see them. In this research, an in situ approach is used to visualize the movement of particulate additives in grease under a shear stress. A model grease in the form of petroleum jelly was mixed with $2.3 w t \% \alpha-$ $\mathrm{ZrP}$ nanoparticles. A custom rotating concentric tube device was built to shear and monitor the grease using tomography technique. Through the use of dual edge micro x-ray computed tomography $(\mu-\mathrm{XCT})$, the distribution of the particulate additives in the Petroleum jelly were visualized. Results showed that prior to shearing, the $\alpha$-ZrP nanoparticles were randomly aggregated throughout the annular gap between the cylinders. However, after a shear stress was applied to the grease mixture the additives were shown to evenly distribute throughout the annular gap. The visual results indicate that the $\alpha-\mathrm{ZrP}$ nanoparticles tend to separate and redistribute along the shearing direction, which attributes to friction reduction. The shear-stress enabled uniformity of particle distribution indicate potential benefits and effectiveness using nanoparticle as additives. This research opens windows for future investigation in lubrication.
\end{abstract}

Keywords: nanoparticles, lubricant additives, in situ detection, 3D visualization, tomography, dispersion, grease

\section{Introduction}

The effects of friction and wear on everyday mechanical systems lead to large drops in efficiency and overall waste in energy. Automobiles, such as cars and trucks, for instance tend to waste an average of $33 \%$ of fuel to overcome frictional losses within the engine, other auxiliary systems $[1,2]$. Proper lubrication within critical areas will cause a reduction in friction and ultimately 
lead to higher efficiently for the entire system. The most common types of lubricants used in mechanical systems are liquid lubricants in the form of oils, and semisolids in the form of greases. Lubricating greases typically behave as a non-Newtonian shear thinning fluid when exposed to a mechanical shear stress [3]. These greases can be broken down into three major components: a base oil, a thickening agent; and additives. The incorporation of organic and inorganic additives in lubricants have been shown to contribute to a reduction in friction and wear within different mechanical systems [4-7]. The performance of an additive relies on several factors including the chemical nature, size, shape, and concentration. The mixing process used to incorporate an additive must also be taken into consideration. The consistency in performance of an additive relies heavily on the homogeneity of the entire mixture $[8,9]$. Decrease in performance can be attributed to an improper mixture, or a mixture that becomes separated over time; de-homogenization $[10,11]$. The manufacturing procedures for grease is therefore a complex process that requires numerous steps and controlled parameters to ensure a consistent product. In addition, grease mixtures will tend to vary in rheological properties under the mechanical stress of the mixing process, with no direct relationship to its performance postprocessing [12]. A new understanding of additive homogenization is needed. Many studies have shown the benefits of incorporating various additives, however there exists a lack of evidence as to how these additive particles interact within the system. In our previous work, we presented a methodology to directly observe additive particles in a grease using X-ray computed tomography (CT) [13]. It was the first time to be able to "see" the particles inside a grease. To further understand how additive particles behavior, the present work will focus on tracking the movement of particle species under shear within a pure base grease.

Computed tomography is a highly versatile technology that is most commonly used for medical purposes to diagnose various ailments within a live subject. It is a nondestructive procedure that can generate two dimensional orthogonal cross sections of an object, as well as full three dimensional models. When utilized under controlled conditions, a synchrotron based micro Xray $\mathrm{CT}(\mathrm{u}-\mathrm{XCT})$ system can produced high resolution images capable of observing objects at a micron and sub-micron scale[14, 15]. Through our past work on grease additives, $\mu$-XCT has been shown to be an effective method for observing particle in grease through the manipulation 
of the monochromatic X-ray energy[13]. The objective of the present research is to expand on the methodology reported previously and study the movement of particles. A simple base grease will be used, and only one particle additive species will be incorporated. The additives to be observed will be $\alpha$-ZrP nanoparticles (NP). The weak van der Waals forces between the two dimensional $\alpha-\mathrm{ZrP}$ platelets has been attributed to the improvement of friction and wear behavior when incorporated into a lubricant [16-18]. Weak bonding forces between each particle allow for them to be easily exfoliated, thereby providing more effective fluid transport for lubrication. The grease mixture will be observed as received and after a controlled shear stress has been applied. Analyzing a pre and post sheared grease under $\mu$-XCT will allow for a better understanding of the particle behavior and the lubrication mechanism.

\section{Methods}

\section{Materials}

The $\alpha-Z r P$ NPs were prepared by the hydrothermal method as reported previously [19]. The lubricant used in this study is petroleum jelly, which is a petroleum based semi-solid. Although not normally used for industrial applications, petroleum jelly is a common lubricant used to moisturize human skin $[20,21]$. It consists primarily of a mineral base oil with various hydrocarbons type thickeners to form a semi-solid, high viscosity fluid. Petroleum jelly exhibits a non-Newtonian shear thinning behavior when under an applied mechanical deformation [22]. Studies have shown petroleum jelly to be a good medium for the analysis of additive particles for friction and wear performance $[23,24]$. For the purpose of this investigation, it will serve as a good medium for the incorporation and study of nanoparticle behavior under shear.

In order to investigate the behavior of the NP additives mixed in the petroleum jelly, two sample groups were studied using a K-edge tomography method; one mixed as received, and one that underwent controlled sliding (shear). Each sample contained Petroleum jelly mixed with Zirconium phosphate $(\alpha-\mathrm{ZrP})$ additive at $2.3 \mathrm{wt} \%$. The petroleum jelly and NPs were simply mixed by hand inside a test tube until a uniform coloration was achieved. The samples and their rotation status are listed in Table 1. The samples were deposited between the inner and outer cylinders of a rotational device that will described in the next section. 
Table 1. List of the two sample groups with their testing conditions.

\begin{tabular}{|c|c|}
\hline Group 1 & Group 2 \\
\hline $\begin{array}{c}\text { Petroleum Jelly + } \\
\text { 2.3wt\% a-ZrP }\end{array}$ & $\begin{array}{c}\text { Petroleum Jelly + } \\
2.3 w \text { \% } \mathrm{a}-Z r P\end{array}$ \\
\hline As received & Sheared \\
\hline
\end{tabular}

\section{Methodology}

In order to observe the grease/additive mixture under controlled sliding conditions, a special testing rig was built. The rig consists of a rotating inner cylinder and a stationary outer cylinder made from polyamide plastic tubes. Polyamide tubes are necessary as they will appear "transparent" under x-ray and allow for an unobstructed view of the grease mixture. The inner cylinder is connected to the shaft of a 2 phase stepper motor and a rotary encoder. The speed of the motor is electronically controlled, while the encoder measures the rotational speed of the inner cylinder. A closed loop control system was used to supply a sufficient amount of torque to maintain a set motor speed over the test period. A model of the rig design is shown in Figure 1.

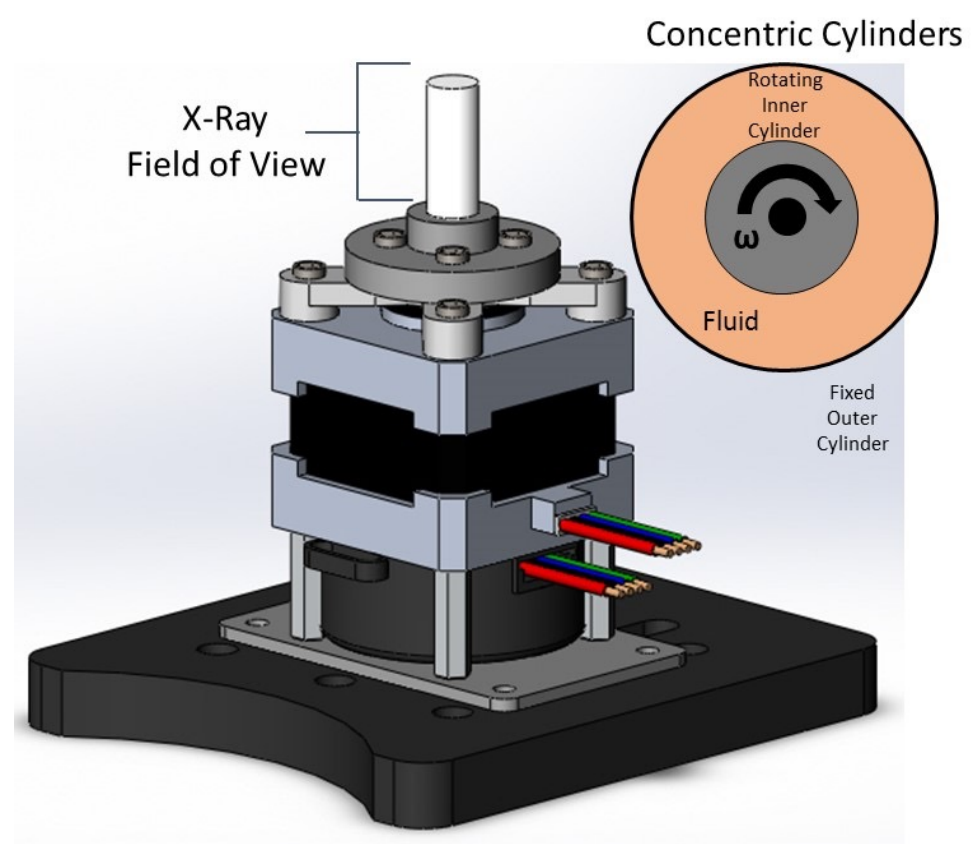

Figure 1. Diagram of the concentric cylinder testing rig used to shear the grease/nanoparticle mixture. 
The entire rig is situated such that the rotational axis of the tubes sit along the central axis of the Beamline sample table as shown in Figure 2. The outer diameter of the stationary outside tube is $4 \mathrm{~mm}$, which is less than the $5 \mathrm{~mm}$ field of vision (FOV) of the microscope used to perform the imaging. This configuration will allow for a uniform scan of the entire annular gap between the cylinders, and a clear image of the grease and NP mixture.

(a)

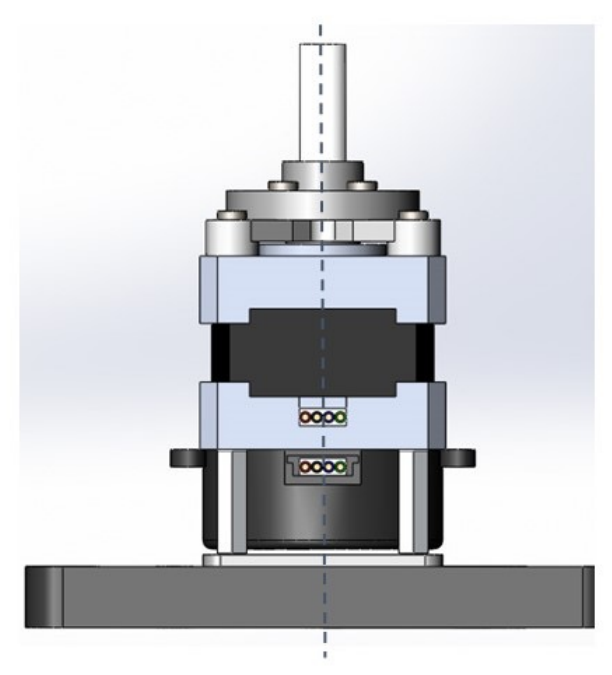

Axis of Rotation (b) $\quad \underline{\text { Top View }}$

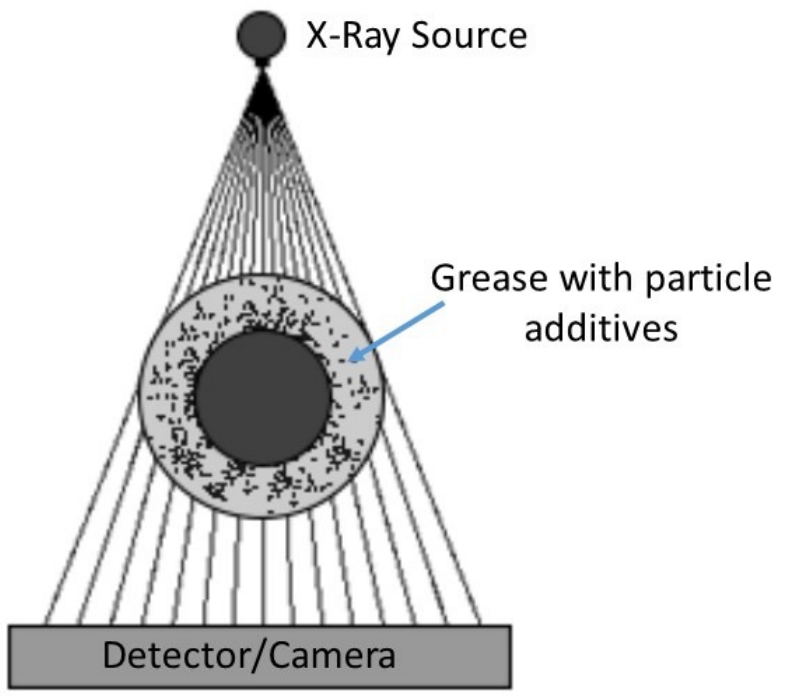

Figure 2. (a) Side view of the testing rig, indicating the axis or rotation, and (b) Top view of the concentric cylinders with grease/additive mixture, illustrating the scanning configuration.

The concentric cylinder device was calibrated prior to placement into the beamline so as to minimize error and simplify the test procedure when placed within the beamline. Based on the specifications of the motor used, a constant torque of $9.5 \mathrm{~N}-\mathrm{cm}$ was established for the grease sample at a rotational speed of 25 revolutions per minute (RPM).

\section{Micro-tomography}

Synchrotron based X-ray micro-tomography was performed at the Advanced Light Source (ALS) facility at Lawrence Berkeley National Laboratory (LBNL). Synchrotron scanning was performed at Beamline 8.3.2 at ALS. Each of the grease samples were scanned at two energy 
levels that correspond to being higher and lower than the K-edge of zirconium (17.998keV). This methodology has been discussed in our previous research elsewhere[13, 14, 25]. The two energies used for the scanning were $17.8 \mathrm{keV}$ and $18.2 \mathrm{keV}$. A 5x optical lens with $0.00129 \mathrm{~mm}$ resolution was used. Other parameter settings and operations were the same as we reported before $[13,14]$.

The distribution of particulate additives in petroleum jelly was visualized using micro x-ray computed tomography. Petroleum jelly was mixed with $2.3 \mathrm{wt} \% \alpha-\mathrm{ZrP}$ nanoparticles and placed between the concentric cylinders of a rotating device to be sheared. Through the use of dual energy K-edge tomography technique, the zirconium element in the particles was distinguishable from the carbon chains comprising the base petroleum jelly grease. Identifying different species within the petroleum jelly mixture allowed for an image mapping of the particulate movement before and after a shear force was applied. The images of the simply mixed petroleum jelly and NPs showed that the particles aggregated in certain areas, a non-homogenous compound. Under the influence of shear, it was observed that the $\alpha-\mathrm{ZrP}$ nanoparticles will tend to separate and redistribute within the annular gap, becoming homogenized. This behavior lends to the reduction in friction coefficient that was reported for petroleum jelly when $\alpha$-ZrP nanoparticles are added. This research discovered the effectiveness of shear-induced homogeneity of nanoparticles that shade the light of low-cost manufacturing of lubricants.

\section{Results}

\section{Localization of additive nanoparticles}

Though use if the dual energy scanning method, the resulting images may be subtracted using image processing to identify individual element species. Radiating elemental species at energies that are above and below their K-edge will cause a change in the mass attenuation coefficient. For this case, the mass attenuation coefficient of the zirconium in the additive $\alpha$-ZrP particles exhibits a sharp increase when subtracting images scanned at $17.8 \mathrm{keV}$ from those scanned at $18.2 \mathrm{keV}$. Image subtraction at these two energies reveals details at an intermediary at the K-edge 
of zirconium $(17.998 \mathrm{keV})$. Other elements in the grease samples, such as carbon in petroleum jelly, were also detected. However, the mass attenuation coefficients for such elemental species exhibited only small values compared to zirconium. Therefore, the additives in the petroleum jelly are clearly distinguishable from the bulk. The absorption coefficient related to each of the scanned elements of the synchrotron x-ray are represented by the pixel values within the reconstructed image datasets. Details of this methodology are presented elsewhere $[13,14,25]$.

To observe the $\alpha-Z r P$ nanoparticles in Group 2, the petroleum jelly samples mixed with 2.3 wt $\%$ $\alpha-\mathrm{ZrP}$ were radiated before and after a controlled shear force was applied. After rotation, the aggregated $\alpha-\mathrm{ZrP}$ additive distributed more uniformly within the annular gap, which will reduce the concentration of the additive overall. The pixel value/absorption coefficient is reduced after shear as well. Comparing the two energies, above $(18.2 \mathrm{keV})$ and below $(17.8 \mathrm{keV})$ the $\mathrm{Zr} \mathrm{K}$ edge, the higher energy datasets have more counts at the same pixel value and larger pixel values than the lower energy datasets. This trend was seen for both grease samples mixed with the $\alpha-$ $\mathrm{ZrP}$ additive.

\section{Distribution of particle additives}

Further study was conducted on pure petroleum jelly. This compound contains a combination of different carbon backbones of various chain lengths. The mass attenuation coefficient of carbon is significantly less than that of zirconium. Hence, by adjusting the image color-map, the $\alpha-\mathrm{ZrP}$ additive can be clearly distinguished from the petroleum jelly. The imaging results are displayed in Figure 3. Figure 3a and Figure 3b show the top view of a 3D visualization for the samples before and after shearing, respectively. Each sample scanned at $18.2 \mathrm{keV}$. Based on the same colormap, the voxel values of the datasets before shear (Figure 3a) are higher than that after shearing (Figure $3 \mathrm{~b}$ ). This indicates that the additives were randomly aggregated within the annular gap before shearing was applied. Once a shear force was applied, the $\alpha$-ZrP additives in the samples became more evenly distributed throughout the annular gap of the concentric tubes. Figure $3 \mathrm{c}$ and Figure $3 \mathrm{~d}$ show the cross-sectional view of the each sample. Figure $3 \mathrm{e}$ and Figure $3 f$ shows the distribution of $\alpha-\mathrm{ZrP}$ additives before and after shear. 


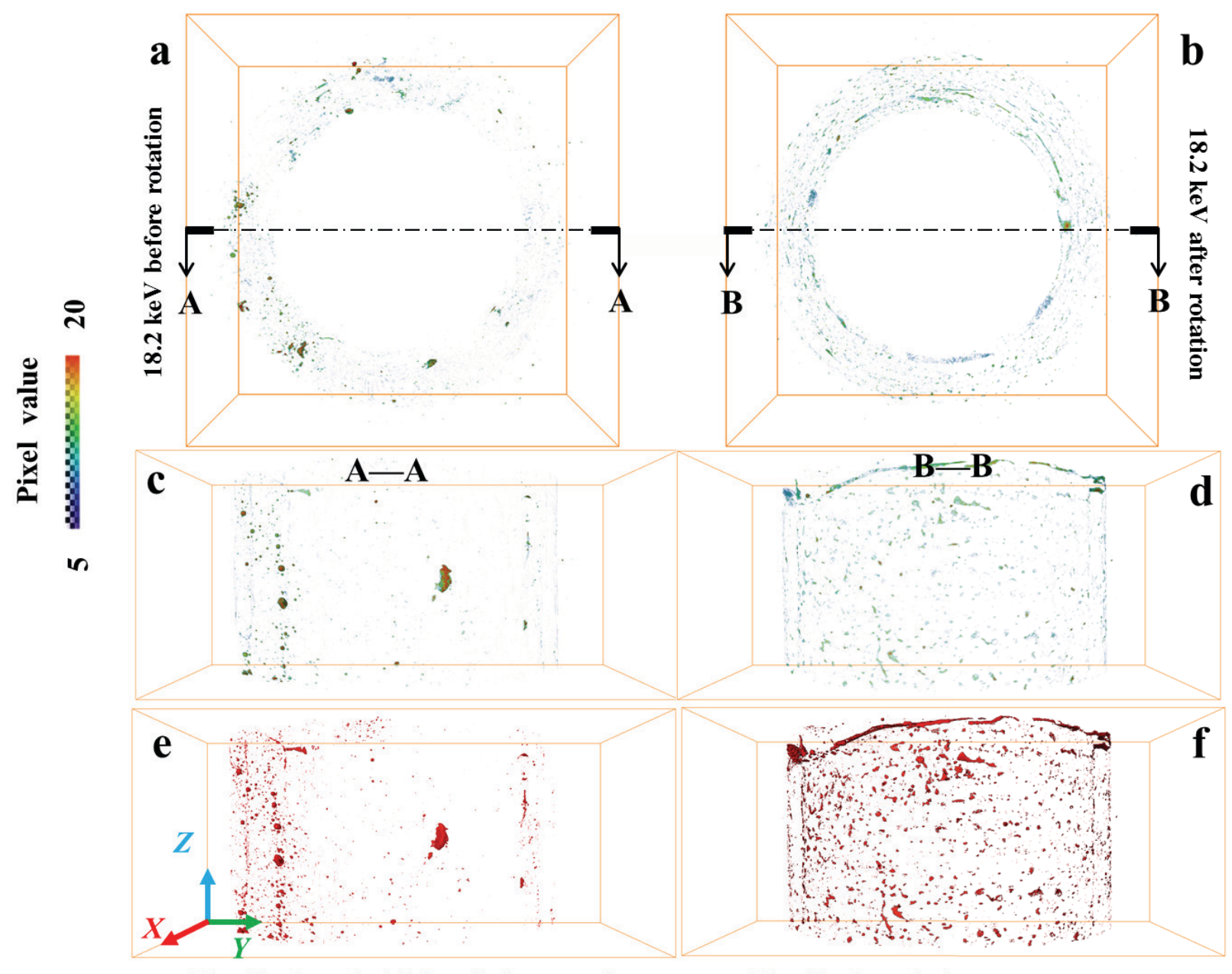

Distribution of additives before rotation

Distribution of additives after rotation

Figure 3. The top view of $3 D$ visualization for group 2 built by $18.2 \mathrm{keV}$ datasets (a) before rotation, (b) after rotation. (c-d) The side sectional view corresponding to the ( $a-b)$. The distribution of $\alpha-Z r P$ additives (e) before rotation and (f) after rotation. The box size is

$$
1.7 \times 3.3 \times 1.5 \mathrm{~mm} \text {. }
$$

As shown in Figure 3, the particles appear to be uniformly distributed when a shear force was applied. We have reported that disk-like nanoparticles of $\alpha-\mathrm{ZrP}$ are effective to reduce friction on oil[26]. Here we discuss about grease.

In order to study the effects of a shear stress on the distribution of $\mathrm{ZrP}$ particles, we deposited the grease with the added particles $(2.3 \mathrm{wt} \%)$ on a glass slice and then slide two for an extended period of time to make sure they are uniformly distributed. The two slices were clamped and placed under an integrated optical microscopy system (MultiView 1000). Observation was 
recorded against time. Results showed that air bubbles were removed in two hours (Figs. 4a,b) and the added particles diffused locally (support information 'Static.gif'). This movement is believed to be driven by the shear force in the grease. Moreover, in order to study the effects of temperature, a heater was used to heat the sample holder to 60 . In just about 4 minutes, the particles were observed to move (Figs. $4 \mathrm{c}-\mathrm{f}$ in 4 mins, marked particles). The larger

the particles, the slower they move. More information can be obtained in the support information as titled 'heat.gif'.
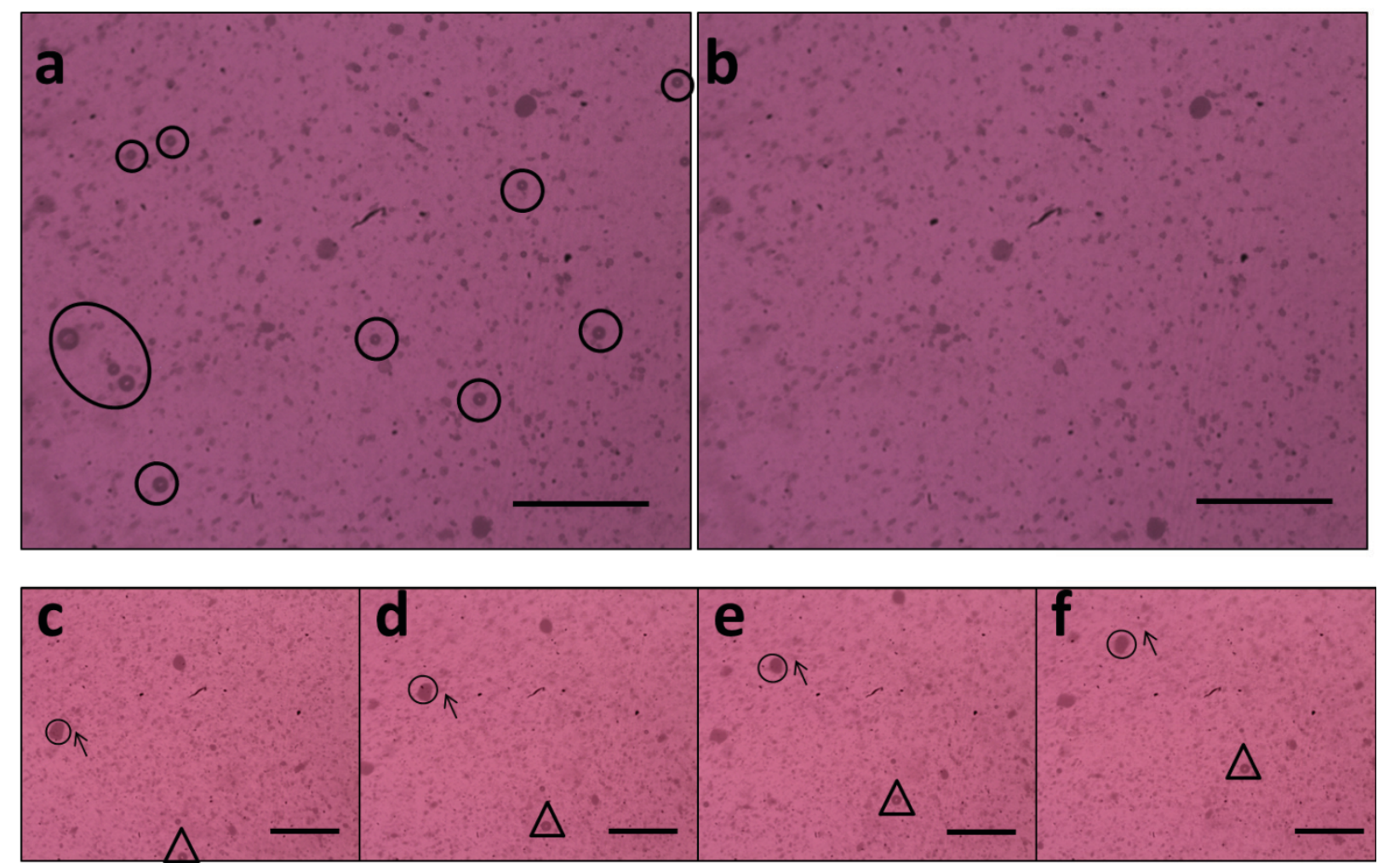

Figure 4. The change of additives in sheared grease under static condition after (a) $0 \mathrm{~h}$ and (b) $2 \mathrm{~h}$. (c-f) The change of additives in sheared grease under 60 in 4 mins. The scale bar is $1 \mathrm{~mm}$.

The frictional performance of the two grease samples were also investigated. Separate tribological tests were conducted under different contact pressures and temperatures using a standard pin-on-disc tribometer. The following figures show the results of friction coefficient over a temperature range of $-100^{\circ} \mathrm{C}$ to $200^{\circ} \mathrm{C}$ for constant contact pressures of $1800 \mathrm{Mpa}$ and 900Mpa. 


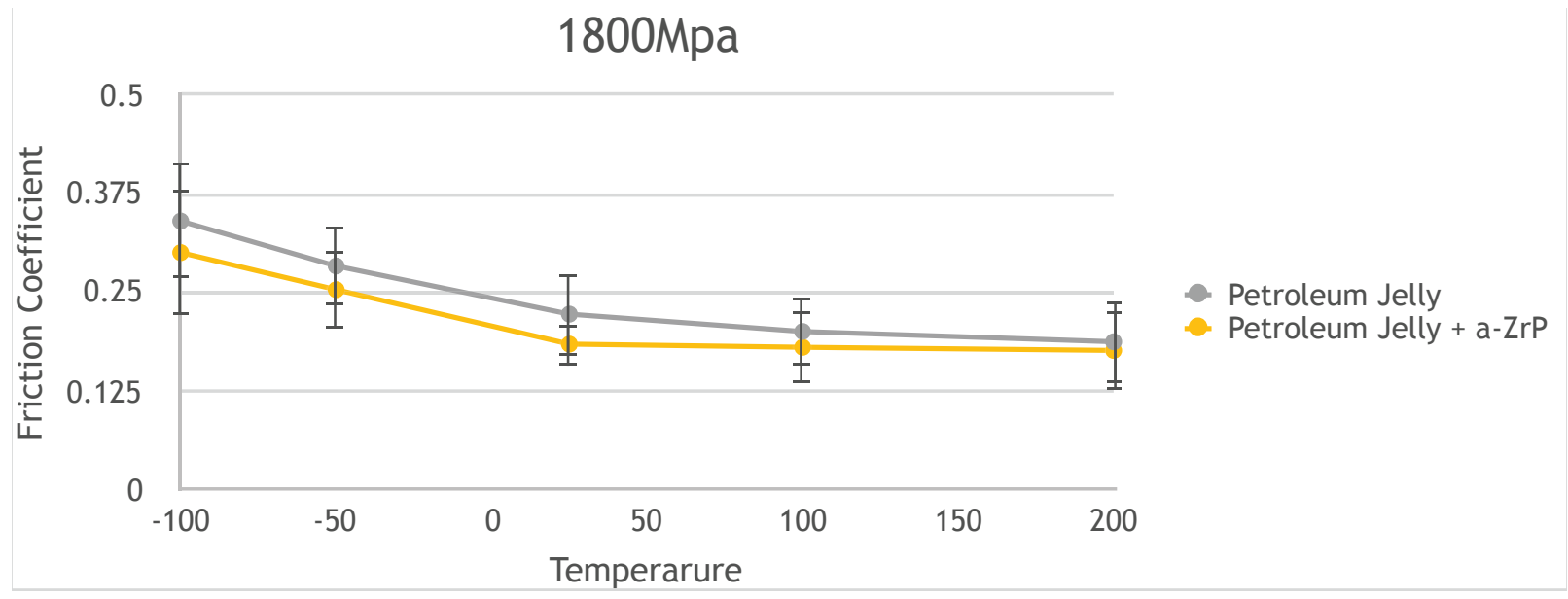

Figure 5. Plot of friction coefficient versus temperature for the two grease groups tested at $1800 \mathrm{Mpa}$ contact pressure.

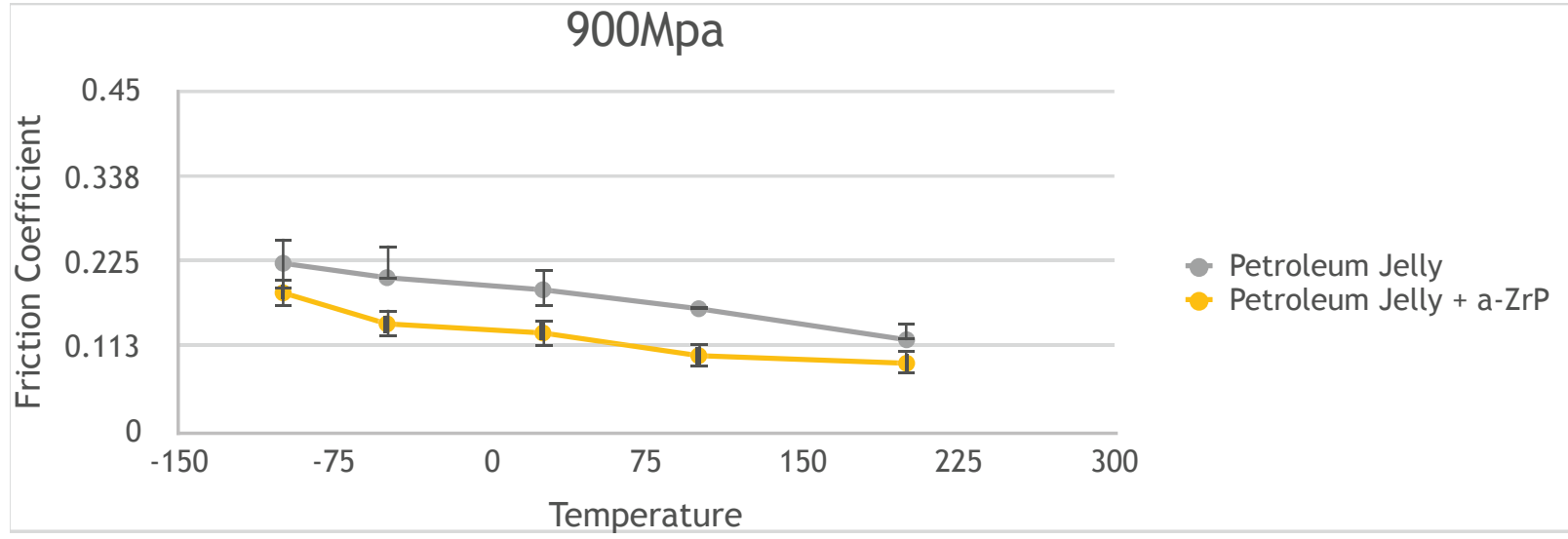

Figure 6. Plot of friction coefficient versus temperature for the two grease groups tested at 900Mpa contact pressure.

As shown in Figures 5 and 6, the two grease samples show a significant difference in friction performance over a wide temperature range at constant contact pressures. The general trend shows that the addition of $2.3 \mathrm{wt} \% \mathrm{aZrP}$ particles will help to improve friction performance over base petroleum jelly. However, at elevated temperatures reaching $200^{\circ} \mathrm{C}$, the difference in performance is not as pronounced. The results at room temperature show the greatest improvement in performance at reduced contact pressure. Therefore, more tests were conducted at room temperature with varying contact pressures to verify these results. The following plot shows the results of the room temperature tests. 


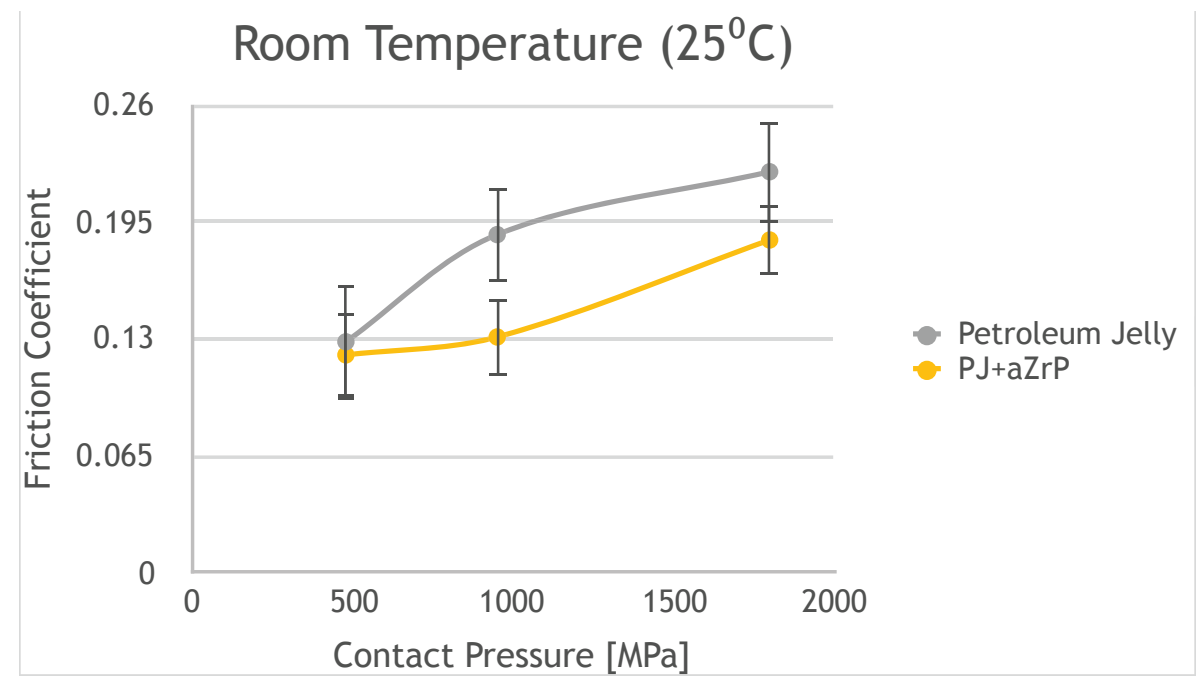

Figure 7. Plot of friction coefficient versus contact pressure for the two grease samples for a constant temperature of $25^{\circ} \mathrm{C}$.

As Figure 7 shows, the greatest improvement in performance occurs at contact pressures around $1000 \mathrm{Mpa}$. However, at lower and higher contact pressures the difference in performance becomes less significant. These results are comparable to the results of the various temperature experiments. The results of the tribological tests shows that there exists optimal conditions for which the introduction of aZrP particles would be beneficial.

\section{Discussion}

\section{Mechanisms of friction reduction}

To understand the behavior of grease, we first estimate the value of the shear stress. The concentric cylinder testing rig was designed based on the mechanics and operation of a standard rotating cylinder viscometer. Based on the geometry and orientation of the testing rig, the diagram shown in Figure 4 was established. 


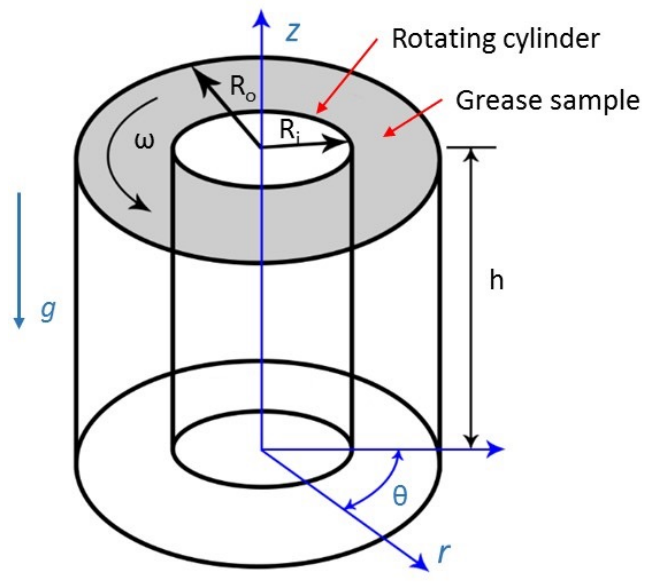

Figure 5. Diagram view of the concentric cylinder testing rig, indicating key parameters in cylindrical coordinates.

For this configuration, the annular gap width will be $0.05 \mathrm{~mm}$. Since the gap is small, and the duration of the test was relatively short, it will be appropriate to neglect the effects of gravity on the grease sample. The shear stress analysis on the grease will follow the Herschel-Bulkley rheological model, which has been shown to be a good approximation for the behavior of petroleum jelly $[22,27]$. The equation of motion of the system can be expressed as:

$$
\rho \frac{D v}{D t}=\rho \frac{\partial v}{\partial t}+\rho(V \cdot \nabla) v=\nabla \cdot \tau+\rho F
$$

where $\frac{D}{D t}$ is the material derivative, $v$ is the velocity vector, $\tau$ is the stress matrix, and $\mathrm{F}$ represents the body forces. As mentioned above, due to the small gap width, the body force of gravity acting on the grease in the axial direction can be neglected. Here, we will assume a one dimensional flow. Hence in cylindrical coordinates, $v=v_{\theta}(r)$ and $v=v_{r}=v_{z}=0$. Due to symmetry in the angular direction, $\frac{\partial}{\partial \theta}=0$. Similarly, the symmetric stress matrix will have nonzero components at $\tau_{\theta \mathrm{r}}$ and $\tau_{\mathrm{r} \theta}$. Equation 1 can be reduced to the form: 


$$
\frac{\partial\left(r^{2} \tau_{\theta r}\right)}{\partial r}=0
$$

Integrating this equation will give the governing momentum equation in the $\theta$-direction as:

$$
\tau_{\theta r}=\frac{C}{r^{2}}=f(r)
$$

where $\tau_{\theta \mathrm{r}}$ is the shear stress, and $\mathrm{C}$ is a constant. A generalized form for Equation 3 for a Newtonian fluid is:

$$
\tau_{\theta r}=\eta(\sqrt{2}) \cdot \frac{d}{d r}\left(\frac{v_{\theta}}{r}\right)
$$

where $\eta$ is the viscosity, and is the shear rate. For a one dimensional flow, which is assumed for our setup, the shear rate can be simplified to the following form:

$$
\sqrt{2} r \frac{d}{d r}\left(\frac{v_{\theta}}{r}\right)
$$

Integrating with respect to the inner and outer radius of the annular gap will yield the equation for the shear rate applied by rotating the inner cylinder. The equation will take the form:

$$
\sqrt{2} \omega \frac{2 R_{i}^{2} R_{o}^{2}}{\left(R_{o}^{2}-R_{i}^{2}\right) R_{a}^{2}}
$$

where $\omega$ is the rotational speed of the inner cylinder, $R_{i}$ is the inner cylinder radius, $R_{0}$ is the outer cylinder radius, and $\mathrm{R}_{\mathrm{a}}$ is the average radius of both cylinders. The shear rate will be determined through the device geometry and the speed of the motor. Greases that show a shear thinning behavior typically follow a power law equation in regards to the shear stress and viscosity. More specifically, our analysis will follow a Hershel-Bulkley rheological model. For this model, the shear stress has the form:

$$
\tau_{\theta r}=\tau_{o}+k(\sqrt{3})
$$


where $\tau_{\mathrm{o}}$ represents a yield stress, $k$ is a consistency factor, and $n$ is the flow index [28]. For the rotating cylinder device, the speed of the inner tube can be controlled and maintained at a constant rate. For this experiment, the inner tube was set to rotate at 50 revolutions per minute (RPM) for one minute to establish a consistent shearing of the grease mixture. The shear rate was found to be $128.17^{\mathrm{s}-1}$. The average shear stress on the petroleum jelly and NP mixture within the annular gap of the concentric cylinders was found to be 136.15Pa. As we know that using Hamaker constant, $10^{-19} \mathrm{~J}$ for a solid particle, the Van der Waals interaction between particles would be in the $\mathrm{nN}$ range [29-32]. Therefore the applied shear force is more than sufficient to separate the adhered particles. These parameters allowed for a uniform shearing of the mixture throughout the annular gap. As shown in the images shown in Figure 3, the aggregated particles observed prior to shear became well distributed through the annular gap after shearing.

To verity the effects of $\alpha-Z r P$ NPs, an ex situ experiment was conducted using a testing procedure commonly used to evaluate the frictional performance and galling resistance of industrial thread compounds [33]. A standard procedure issued by the American Petroleum Institute (API) is used to evaluate the petroleum jelly and NP mixture. The friction factors for pure petroleum jelly and petroleum jelly + NPs were 0.64 and 0.73 , respectively. The friction coefficients were found to be 0.125 and 0.081 for petroleum jelly, and petroleum jelly + NPs, respectively. Thus, the incorporation of $\alpha$-ZrP nanoparticles into petroleum jelly resulted in a reduction of friction.

This research used the tomography technique to observe nanoparticles in a grease under a shear stress. We found that $\alpha-\mathrm{ZrP}$ nanoparticles became uniform after a shear force is applied. Stress analysis and ex situ experiments confirmed that nanoparticles effectively carried load to reduce friction. The finding is summarized in Figure 5. 
(a) Grouping of nanoparticles

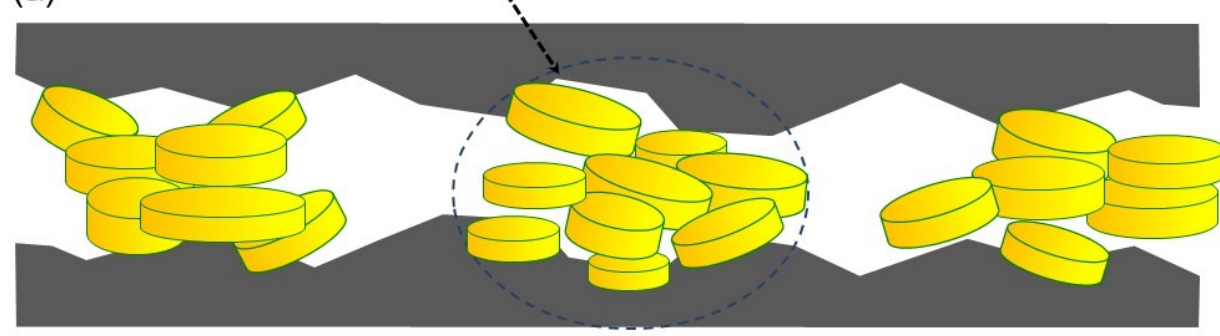

(b)

Sliding

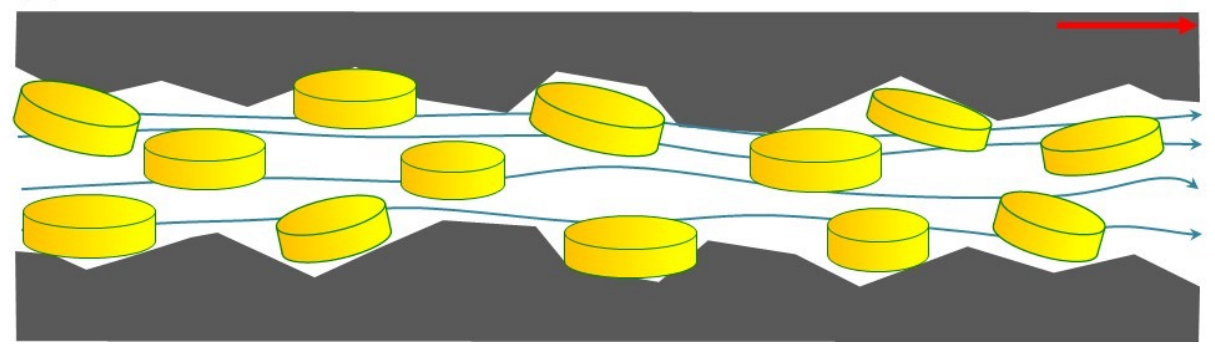

Figure 6.(a) Non-homogenous grouping of nanoparticles, and (b) Uniform distribution achieved through shearing.

Our results and discussion above have proven that incorporating $\alpha$-ZrP NPs into petroleum jelly can significantly improve frictional performance. The reason of friction reduction is due to the uniformly distributed nanoparticles that effectively carry a load. It has been accepted that the weak van der Wall forces between each particle caused them to become easily exfoliated under shear, thereby enhancing lubrication[26]. The methodology presented herein allowed for a visual confirmation that $\alpha-Z r P$ nanoparticles become re-distributed under mechanical deformation. The uniform distribution can be attributed to the particles readily exfoliating under shear.

\section{Acknowledgements}

To be provided once the manuscript is accepted.

\section{References}

1. Holmberg, K., P. Andersson, and A. Erdemir, Global energy consumption due to friction in passenger cars. Tribology International, 2012. 47: p. 221-234. 
2. Holmberg, K., et al., Global energy consumption due to friction in trucks and buses. Tribology International, 2014. 78: p. 94-114.

3. Martin, J.M. and N. Ohmae, Colloidal Lubrication: General Principles, in Nanolubricants. 2008, John Wiley \& Sons, Ltd. p. 1-13.

4. Li, B., et al., Tribochemistry and antiwear mechanism of organic-inorganic nanoparticles as lubricant additives. Tribology Letters, 2006. 22(1): p. 79-84.

5. Gorbatchev, O., et al., Friction reduction efficiency of organic Mo-containing FM additives associated to ZDDP for steel and carbon-based contacts. Tribology International, 2016. 99: p. 278-288.

6. Ping-Hsueh Tsai, m.m.y.c.t. and h.m.k.e.t. Hsiao-Yeh Chu, Effects of the NanoDiamond Additives on the Tribological Performance Improvement of Lubricating Grease. Key Eng. Mater., 2015. 642: p. 298-302.

7. Jatti, V.S. and T.P. Singh, Copper oxide nano-particles as friction-reduction and antiwear additives in lubricating oil. Journal of Mechanical Science and Technology, 2015. 29(2): p. 793-798.

8. Lukkassen, D., A. Meidell, and P. Wall, On the Role of Homogenization in Lubrication Theory. AIP Conference Proceedings, 2014. 1637(1): p. 1170-1176.

9. Alisin, V.V., et al., Studying colloidal greases with hard nanoparticles of aluminum silicates used to reduce wear of steel friction pairs. Vol. 34. 2013: Journal of Friction and Wear. 6.

10. Deeva, V.S.v.m.r., S.M.s.n.r. Slobodyan, and V.S. Teterin, Optimization of Oil Particles Separation Disperser Parameters. Mater. Sci. Forum, 2016. 870: p. 677-682.

11. Mel'nik, D.T., et al., Influence of homogenization conditions on properties of bentonite greases. Chem. Technol. Fuels Oils, 1986. 22(2): p. 84-86.

12. Franco, J.M., et al., Mixing rheometry for studying the manufacture of lubricating greases. Chem. Eng. Sci., 2005. 60(8-9): p. 2409-2418.

13. Chen, Y., et al., Direct observation of lubricant additives using tomography techniques. Appl. Phys. Lett., 2016. 109(4).

14. Chen, Y., et al., Erratum to: Observation of yttrium oxide nanoparticles in cabbage (Brassica oleracea) through dual energy K-edge subtraction imaging. Journal of Nanobiotechnology, 2016. 14(1).

15. Biziks, V., et al., Assessment of wood microstructural changes after one-stage thermohydro treatment (THT) by micro X-ray computed tomography. Holzforschung: International Journal of the Biology, Chemistry, Physics, \& Technology of Wood, 2016. 70(2): p. 167-177.

16. He, X., et al., a-Zirconium phosphate nanoplatelets as lubricant additives. Colloids Surf. Physicochem. Eng. Aspects, 2014. 452: p. 32-38.

17. Xiao, H.P., et al., Amine-intercalated alpha-zirconium phosphates as lubricant additives. Appl Surf Sci, 2015. 329: p. 384-389.

18. Yue, Y., et al., Correlating hydrodynamic radii with that of two-dimensional nanoparticles. Applied Physics Letters, 2015. 107(25).

19. He, X.L., et al., Two-dimensional nanostructured Y2O3 particles for viscosity modification. Appl. Phys. Lett., 2014. 104(16).

20. Gold, M.I., Use of petroleum jelly. Anesthesiology, 1985. 63(3): p. 339-40.

21. Bigby, K.M., Apply petroleum jelly to lesions before treatment. Fam. Pract. Manag., 2010. 17(6).

22. Park, E.K. and K.W. Song, Rheological Evaluation of Petroleum Jelly as a Base Material in Ointment and Cream Formulations: Steady Shear Flow Behavior.

23. Oganesova, E., et al., Synthesis of tungsten sulfide nanoparticles and their tribological properties as additives for lubricating oils. Nanotechnologies in Russia, 2016. 11(5/6): p. 312.

24. Robinson, T., H. Ou, and C.G. Armstrong, Study on ring compression test using physical modelling and FE simulation. J. Mater. Process. Technol., 2004. 153-154: p. 54-59. 
25. Chen, Y., et al., Observation of two-dimensional yttrium oxide nanoparticles in mealworm beetles ( Tenebrio molitor). Journal of Synchrotron Radiation, 2016. 23(5): p. 1197-1201.

26. He, X., et al., Two-dimensional nanostructured Y2O3 particles for viscosity modification. Appl. Phys. Lett., 2014. 104(16): p. 163107.

27. Lacaze, L., A. Filella, and O. Thual, Steady and unsteady shear flows of a viscoplastic fluid in a cylindrical Couette cell. Journal of Non-Newtonian Fluid Mechanics, 2015. 220: p. 126-136.

28. Al-Zahrani, S.M., A generalized rheological model for shear thinning fluids. Journal of Petroleum Science and Engineering, 1997. 17(3): p. 211-215.

29. Hamaker, H.C., The London-van der Waals attraction between spherical particles. Physica, 1937. 4(10): p. 1058-1072.

30. Bradley, R.S., LXXIX. The cohesive force between solid surfaces and the surface energy of solids. The London, Edinburgh, and Dublin Philosophical Magazine and Journal of Science, 1932. 13(86): p. 853-862.

31. Kokkoli, E. and C.F. Zukoski, Interaction Forces between Hydrophobic and Hydrophilic Self-Assembled Monolayers. J. Colloid Interface Sci., 2000. 230(1): p. 176-180.

32. de Boer, J.H., The influence of van der Waals' forces and primary bonds on binding energy, strength and orientation, with special reference to some artificial resins. Transactions of the Faraday Society, 1936. 32(0): p. 10-37.

33. API Recommended Practice on Thread Compounds for Casing, Tubing, and Line Pipe. API Recommended Practice on Thread Compounds for Casing, Tubing, and Line Pipe. 2003/R2015. 